\title{
Differentially Private Generative Adversarial Networks with Model Inversion
}

\author{
Dongjie Chen \\ University of California \\ Davis, CA \\ cdjchen@ucdavis.edu
}

\author{
Sen-ching Samson Cheung \\ University of Kentucky \\ Lexington, $\mathrm{KY}$ \\ sccheung@ieee.org
}

\author{
Chen-Nee Chuah \\ University of California \\ Davis, CA \\ chuah@ucdavis.edu
}

\author{
Sally Ozonoff \\ University of California \\ Davis, CA \\ sozonoff@ucdavis.edu
}

\begin{abstract}
To protect sensitive data in training a Generative Adversarial Network (GAN), the standard approach is to use differentially private (DP) stochastic gradient descent method in which controlled noise is added to the gradients. The quality of the output synthetic samples can be adversely affected and the training of the network may not even converge in the presence of these noises. We propose Differentially Private Model Inversion (DPMI) method where the private data is first mapped to the latent space via a public generator, followed by a lower-dimensional DP-GAN with better convergent properties. Experimental results on standard datasets CIFAR10 and SVHN as well as on a facial landmark dataset for Autism screening show that our approach outperforms the standard DP-GAN method based on Inception Score, Frechet Inception Distance, and classification accuracy under the same privacy guarantee.
\end{abstract}

Index Terms-Generative adversarial networks, differential privacy, model inversion

प

\section{INTRODUCTION}

With the advances in digital information collection techniques, governments and private enterprises can easily collect sensitive information about anyone in the society. As such, database privacy has become an important topic for legal, ethical, and technical reasons. According to [1], database privacy can be achieved via one of two paradigms. In an interactive setting, a data curator collects data from individuals and provides privacy preserving interfaces for analysts to query data. In a non-interactive setting, a curator releases a "sanitized" or "synthetic" version of data to protect privacy and ensure utility. After the data is released, the curators will not modify the data again and may delete the original data. In the age of deep learning, a common way for non-interactive data release is to generate synthetic data using a Generative Adversarial Networks (GANs) trained on the original private data.

GANs have gained significant popularity since its invention in [2] and have been used in many applications including image-to-image translation [3], face image synthesis [4], and image inpainting [5]. As GANs gain popularity, there

Best Student Paper Award of 13th IEEE International Workshop on Information Forensics and Security (WIFS 2021), Montpellier, France. (C) 2021 IEEE.

DOI: 10.1109/WIFS53200.2021.9648378 are also increasing privacy concerns on whether GANs can compromise the privacy of the original training data [6], particularly if they are used for private data release such as medical data sharing [7]. Attack vectors on GANs such as membership inference attacks [8] and full reconstruction [9] have been investigated. As such, it is imperative to develop robust privacy protection schemes for GANs.

The de facto approach to provide privacy guarantee in machine learning is through the differential privacy (DP) framework [1]. Two different DP schemes have been proposed for GANs: Private Aggregation of Teacher Ensembles (PATE) and Differentially Private Stochastic Gradient Descent (DPSGD). PATE-GAN, proposed in [10], achieves differential privacy by training multiple teacher discriminators using disjoint parts of the training set and one student discriminator. The requirement of using many teacher discriminators trained on different parts of the training set makes PATE-GAN challenging to scale to high-dimensional datasets with limited number of training samples [11].

DPSGD, originally proposed in [12], aims at making the SGD process differentially private by clipping and adding random noise to the aggregated gradients. The usage of DPSGD goes beyond GAN and has been applied to many SGD-based deep-learning techniques [13]-[16]. The unique challenge of DPSGD-based GANs is its difficulty in training - due to the game-theoretic formulation of the cost function, the training process of a complicated GAN does not necessarily converge or converges to a noisy equilibrium, resulting in mode collapse and/or poor synthetic sample quality [6]. A common remedy is to repeat the training process until convergence or depletion of the privacy budget [17], but such a method does not necessarily help the convergence of the training process. As such, DPSGD has not been widely tested on high dimensional multimedia datasets beyond simple test cases such as MNIST.

In this paper, we propose a novel approach called Differentially Private Model Inversion (DPMI) to improve DPSGDbased GANs. Due to the difficulty in training complex DPGANs, DPMI first trains the same high-dimensional GAN (latent dimensional space $\mathbb{R}^{d}$ ) but using only public data, thereby negating the need of using any DP noise. To align with the statistics of the private data, we use the trained generator $G_{p}$ and identify a group of private latent vectors $z_{s} \in \mathbb{R}^{d}$ 
To provide a DP mechanism for training of a deep network on private data, the most straightforward approach is to use DPSGD [13], of which the key steps are summarized below:

1) Randomly sample a mini-batch of private training data and compute the empirical loss and gradients.

2) The magnitude of the gradient function for each training sample is clipped at a clipping bound $d$.

3) Based on the target $\epsilon$ and $\delta$, a privacy accountant mechanism such as [21] determines the number of iterations and the variance of independent zero-mean Gaussian noise to be added to the aggregate gradient.

4) Update the weights of the network using the noisy gradient until the number of iteration hits the privacy budget.

Note that DPSGD is applied only during the training of the discriminator as the generator does not have any access to the private training data.

\section{PROPOSED DPMI FRAMEWORK}

Figure 3 shows the data release process of our proposed DPMI framework. In the public domain, a GAN is trained on publicly available image data $D_{p}$. The resulting trained generator $G_{p}$ is used in the private domain where a model inversion process, detailed in Section III-B, is performed to find the latent vector $z_{s}$ that can best approximate, via $G_{p}$, each image from the private database $D_{s}$. A lower-dimensional DP-GAN is then trained on this collection of latent vectors. The resulting DP generator $G_{d s}$ is used to publicly release a database of synthetic latent vectors that can be used to synthesize synthetic images with $G_{p}$. The privacy of this DPMI framework is discussed in Section III-B.

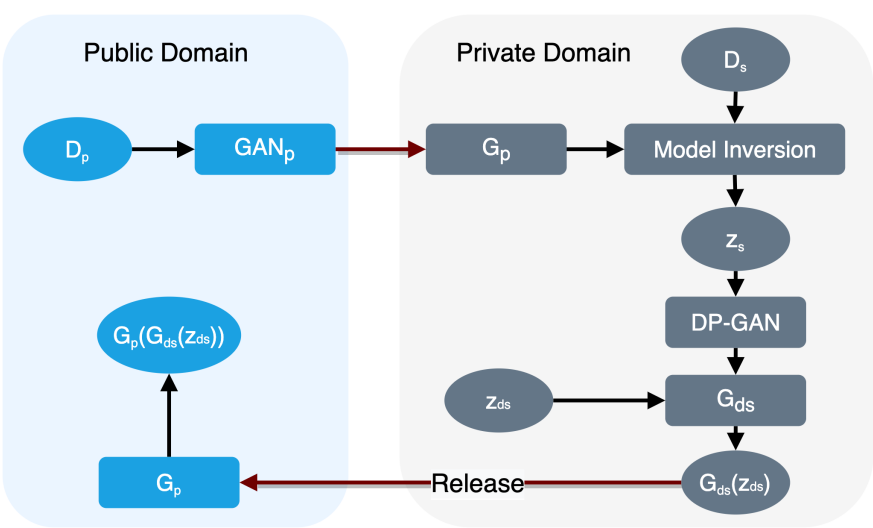

Fig. 3. Proposed DPMI Framework

\section{A. Model Inversion of GANs}

By training a GAN using only public data, there is no need to incorporate any DP mechanisms. This greatly mitigates the convergent problem brought on by the DP noise added to the training process. Due to the differences between the public and private images, the resulting generator will not be able to synthesize images similar to the private images. Nevertheless, with sufficient diversity in the public data, this public generator should be general enough to approximate private images given the "right" latent vectors. We term the process of identifying these latent vectors: model inver sion. Unlike model inversion attacks [9] that are designed to learn private training data given a trained model, our model inversion process has full access to the private data and is performed entirely within the private domain.

Given a trained public generator $G_{p}$ that maps a random $d$-dimensional latent vector $z \sim P_{Z}=\mathcal{N}^{d}(0, I)$ to a synthetic image $G_{p}(z)$, our goal is to identify, for each private image $x_{s} \in D_{s}$, the latent vector $z_{s}$ that minimizes the mean square difference between $x_{s}$ and $G_{p}\left(z_{s}\right)$. More specifically, the model inversion solves the following optimization process:

$$
\begin{aligned}
z_{s}=\underset{z}{\arg \min } & \left\|G_{p}(z)-x_{s}\right\|^{2} \\
\text { s.t. } & P_{Z}(z) \geq P_{Z}\left(z_{0}\right) \text { with } z_{0} \sim P_{Z}
\end{aligned}
$$

We start the model inversion process by first drawing a random sample $z_{0}$ from $P_{Z}$ and the constraint in (4) is to ensure that the quality of $G_{p}\left(z_{s}\right)$ to be comparable to that of $G_{p}\left(z_{0}\right)$. This convex optimization can be easily solved via stochastic gradient ascent procedure by projecting the search trajectory back onto the convex constraint [22].

\section{B. Differentially private GAN on the latent space}

With a well-trained and generalizable $G_{p}$, the model inversion can identify $z_{s} \in \mathbb{R}^{d}$ that can yield very good reconstruction of private images, even if there is no overlap in classes between $D_{p}$ and $D_{s}$ like the one shown in Figure 1. On the other hand, it is very difficult to make the model inversion process differentially private. The model inversion process takes a single image as the input database, which means that we must consider the significant increase in MSE when using an empty set as a neighboring database. This implies that a substantial amount of noise must be added to obfuscate between these two neighboring databases.

In DPMI, we have adopted an alternative approach - we treat the entire collection of $z_{s}$ as the surrogate of $D_{S}$ and train a DP-GAN to synthesize latent vectors that closely resemble the private $z_{s}$ 's. Unlike the image-based DP-GAN, this latent space DP-GAN is of substantially lower dimension with a very well-defined range space as they are all originated from $\mathcal{N}^{d}(0, I)$. As demonstrated in [18], a lower-dimensional DPGAN is easier to train with far better convergent properties. The resulting generator $G_{d s}$ maps a $l$-dimensional $(l<d)$ latent vector $z \in \mathbb{R}^{l}$ to a $d$-dimensional latent vector. For public data release, we randomly sample $z \sim \mathcal{N}^{l}(0, I)$ and synthesize synthetic image $G_{p}\left(G_{d s}(z)\right)$. This whole process inherits the DP property from the DP-GAN, and the subsequent step of $G_{p}$ is a post-processing step that does not alter the DP property of the input, according to Theorem 1 . As such, the DPMI framework is $(\epsilon, \delta)$-DP if the latent space DP-GAN is $(\epsilon, \delta)$-DP, which was demonstrated in [12]. 


\section{EXPERIMENTS}

In this section, we perform an ablation study on the model inversion, and compare the performances between the proposed DPMI scheme and DP-GAN at different privacy levels. To measure the performance of a GAN, we train a classifier using the output synthetic data and measure its accuracy on a hold-out test set of real images, as well as the quality of the synthetic images using established image quality and diversity metrics. This combined assessment approach directly measures the validity of DPMI as a privacy-preserving data release mechanism for public machine learning.

\section{A. Dataset Partition}

To make our proposed scheme broadly applicable to any GANs, we do not rely on any conditional inputs to the training. As such, the generated synthetic data do not have labels and we need to extract a labeling dataset from our training dataset to train a labeling classifier. For all our experiments, we separate the dataset properly into public and private domains. As the public dataset should be sufficiently different from the private dataset, we partition the dataset vertically, setting half of the classes as private and the other half as public.

we first randomly select one-third of the training set as the labeling dataset $D_{l}$, which is used to train the labeling classifier. Then we divide the rest of the training set equally into $D_{p}$ and $D_{s}$, with a random half of the classes goes to $D_{p}$ and the other half goes to $D_{s} . D_{p}$ is treated as the public training set to train the public GAN and $D_{s}$ as the private training set is used for the model inversion process. The holdout testing set contain test images from private classes only so as to test how well a classifier can predict private class labels.

\section{B. Datasets and Evaluation Metrics}

Three datasets are used in our experiments:

1) CIFAR10 [23] contains 50,000 training and 10,000 testing color natural images in 10 classes of size $32 \times 32 \times 3$. In splitting between the public and private training sets, we randomly chose automobile, bird, cat, deer, and dog for $D_{p}$, and frog, horse, ship, truck, and airplane for $D_{s}$.

2) SVHN [24] is a color door-sign digit image dataset, containing 73,257 training images and 26,032 testing images of size $32 \times 32 \times 3$. The public set $D_{p}$ contains digits $1,5,7,8$ and 9 and $D_{s}$ contains digits $0,2,3,4$ and 6.

3) Autism Spectrum Disorder Face (ASDF) dataset is a collection of children's facial landmark features extracted from videos captured for ASD screening [25]. The dataset has 2,675,540 299-dimensional feature vectors consisting of facial landmarks, head pose, eye gaze and facial action units. Our target classification task is smile detection and the dateset is partitioned into 2,160,093 training and 515,447 testing samples. For the public/private split, we randomly separate the training set and use half of it as $D_{p}$ and use another half of it as $D_{s}$.
As these datasets post varying degrees of difficulty in their classification, different classification networks are used. For CIFAR10 and SVHN, we use convolutional neural network (CNN) classifiers with four convolution and max-pooling layers. For ASDF, we use a hybrid structured classifier, containing both CNN and fully-connected layers.

For performance measurements, we consider the accuracy of the trained classifier on the hold-out datasets, as well as image quality and diversity of the synthetic images. For CIFAR 10 and SVHN, we use accuracy as the metric for classification performance as both datasets are balanced. The classification target is to predict the output labels for synthetic images. The ASDF dataset is an imbalanced dataset so we use the macro averages of precision, recall and F1-score as the evaluation metrics. For image quality and diversity measurements, we use Inception Score (IS) and Frechet Inception Distance (FID) based on a classifier trained on ImageNet to measure both synthetic image quality and diversity [26]. These evaluation metrics are used only on CIFAR10 and SVHN as ASDF is not an image dataset.

\section{Ablation study on Model Inversion}

To show the effectiveness of model inversion, we first evaluate model inversion under an ablation study. In this study, we first trained two non-DP GANs with generators $G_{s}$ and $G_{p}$ on the private and public training datasets $D_{s}$ and $D_{p}$ respectively. Three image classifiers are constructed based on the original private dataset $D_{s}$, synthetic images generated by $G_{s}$, and synthetic images generated through the model inversion process on the generator $G_{p}$. The test accuracy of classifiers trained on the image datasets are shown in Table II The macro-averages of precision, recall and F1score of classifiers trained on the ASDF dataset are shown in Table 凹

TABLE I

TEST ACCURACY OF CLASSIFIERS TRAINED ON $D_{s}$, SYNTHETIC DATA GENERATED BY $G_{s}$, AND $G_{p}$ WITH MODEL INVERSION

\begin{tabular}{|c|c|c|c|}
\hline Dataset & $D_{s}$ & $G_{s}$ & $G_{p}+$ Model Inversion \\
\hline CIFAR10 & 0.87 & 0.75 & 0.76 \\
SVHN & 0.92 & 0.84 & 0.92 \\
\hline
\end{tabular}

TABLE II

TEST MACRO AVERAGE RESULTS OF CLASSIFIERS TRAINED ON $D_{s}$, SYNTHETIC DATA GENERATED BY $G_{s}$, AND $G_{p}$ WITH MODEL INVERSION FOR ASDF DATASET

\begin{tabular}{|c|c|c|c|}
\hline & precision & recall & f1-score \\
\hline$D_{s}$ & 0.66 & 0.69 & 0.67 \\
$G_{s}$ & 0.63 & 0.67 & 0.63 \\
$G_{p}+$ Model Inversion & 0.65 & 0.68 & 0.66 \\
\hline
\end{tabular}

Tables II and III show that for both SVHN and ASDF, model inversion is able to achieve much better classification performance than synthetic images and rival those trained using the real private data. For CIFAR10, the gain is not as dramatic due to the relatively simplistic structure of our 
WGAN network. To further examine how dissimilar the public and private datasets can be, we focus on the classification task on SVHN, and use CIFAR10 as the public $D_{p}$ and SVHN as the private $D_{s}$. In this experiment, the entire training and test datasets are used without any partitions. The same three configurations as the previous experiment are used and the results are shown in Table [II] Model inversion method has a seven percentage point higher accuracy than using a private GAN and only a three percentage point lower than using the real SVHN training images.

TABLE III

TEST ACCURACY OF CLASSIFIERS TRAINED ON SYNTHETIC DATA WITH SVHN AS $D_{s}$ AND CIFAR 10 AS $D_{p}$

\begin{tabular}{|c|c|}
\hline Method & Accuracy \\
\hline$D_{s}$ & 0.96 \\
SVHN trained $G_{s}$ & 0.86 \\
CIFAR10 trained $G_{p}+$ Model Inversion & 0.93 \\
\hline
\end{tabular}

\section{Comparison with DP-GAN}

In this section, we compare the DPMI scheme with the DPGAN implementation from [13]. Specifically, we compare the accuracy of the classifiers trained using the synthetic data from DPMI and DP-GAN, and use the synthetic data generated by the private $G_{s}$ as the idealistic upper bound.

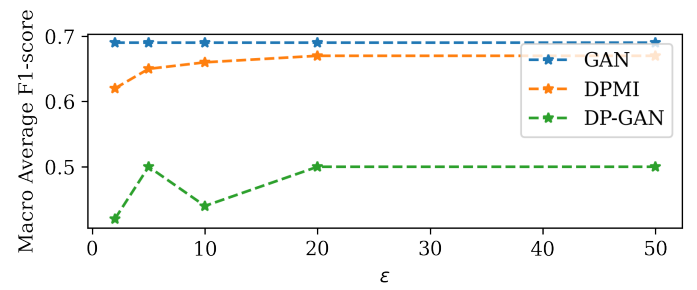

Fig. 4. The F1-score of the classification for ASDF with different $\epsilon$.

Figures 4 and 5 show the trade-off between testing accuracy and the privacy level $\epsilon$ for the three datasets. We find accuracy improvements from $34 \%$ ( 0.75 vs $0.41, \epsilon=50$ ) to $39 \%$ ( 0.67 vs $0.28, \epsilon=10$ ) on CIFAR10. For SVHN, our method has improvements from $33 \%$ ( 0.76 vs $0.43, \epsilon=50)$ to $36 \%(0.75$ vs $0.39, \epsilon=20)$. For ASDF, our method has improvements from $15 \%(0.65$ vs $0.50, \epsilon=5)$ to $22 \%$ (0.66 vs $0.44, \epsilon=10)$. In fact, as shown Figure 4 . DPMI achieves similar performance as the ideal situation while DP-GAN simply does not converge as the results are mostly random guess on the smile/non-smile detection.

Next, we consider the image quality and diversity among all the synthetic images. In Figure 7 and 8 , we compare the DPMI's performance to DP-GAN using Inception Score (IS) and Frechet Inception Distance (FID). A larger IS indicates the synthetic images have better quality and diversity. Figure 7 shows that DPMI has higher IS than DP-GAN when they guarantee the same amount of privacy for both CIFAR10 and SVHN. FID is based on the feature distance between synthetic

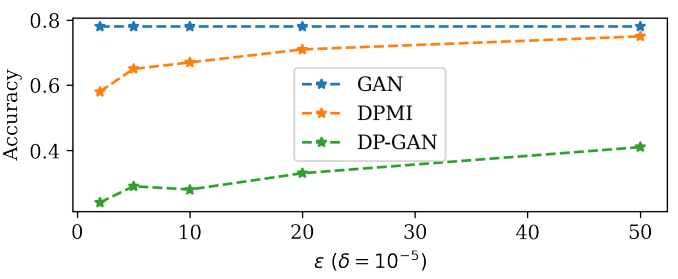

(a) CIFAR10

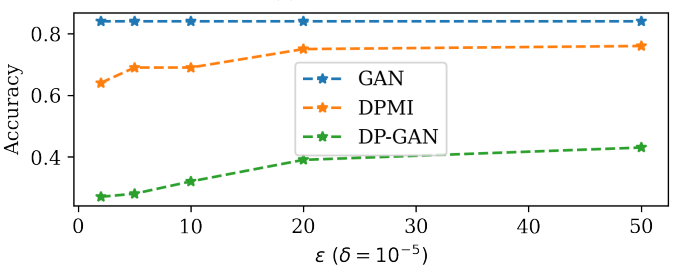

(b) SVHN

Fig. 5. The accuracy of the classification task for CIFAR10 and SVHN with different $\epsilon$.

images and real testing images with the same labels. A smaller FID value means the synthetic images are closer to the real ones. As shown in Figure 8, DPMI has smaller FID values than DP-GAN under the same $\epsilon$ for CIFAR10 and SVHN. Note that since our private datasets only contains images from half of all the classes, the IS and FID values are generally worse than the state-of-the-art results. However, the relative comparisons using IS and FID sufficiently demonstrate that DPMI has better performance than the DP-GAN under the same privacy guarantee.

As ASDF is not an image dataset, we are unable to compute neither IS nor FD. Figure 6 shows a few visual examples from the original dataset and the synthetic ones from both DP-GAN and DPMI using $\epsilon=10$. Note that only the facial landmarks are displayed while the rest of the feature vectors do not have any reasonable methods for visualization. Nevertheless, it is quite clear that DPMI is able to produce reasonable landmarks while DP-GAN produces random results due to poor convergence of the training.

\section{COnClusions}

In this paper, we have introduced DPMI, a new differentially private generative framework of releasing synthetic private data by applying model inversion to map the real private data to the latent space via a public generator, followed by a lower-dimensional DP-GAN. Using two standard image datasets and one privacy-sensitive medical dataset, we have shown that model inversion process can improve the quality of the synthetic images and the lower-dimensional DP-GANs are able to produce better performance than the state-of-theart DP-GAN in classification performance, synthetic image quality and diversity. Further investigation will focus on how different structures of GANs would affect the DPMI process and the end-to-end training of the model inversion process and the low dimensional DP-GAN. 


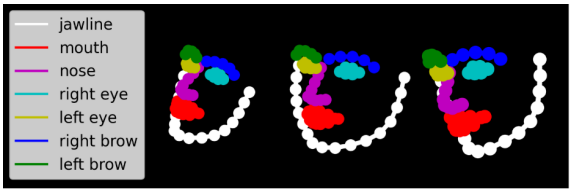

(a) real

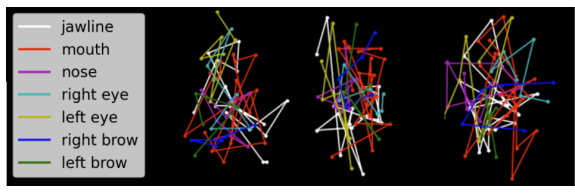

(b) DP-GAN ( $\epsilon=10)$

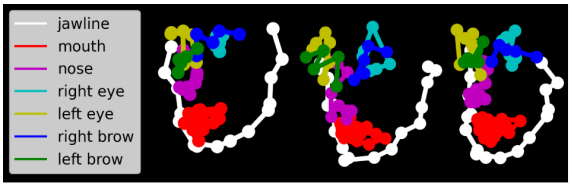

(b) DPMI $(\epsilon=10)$

Fig. 6. Visual Results on ASDF dataset.
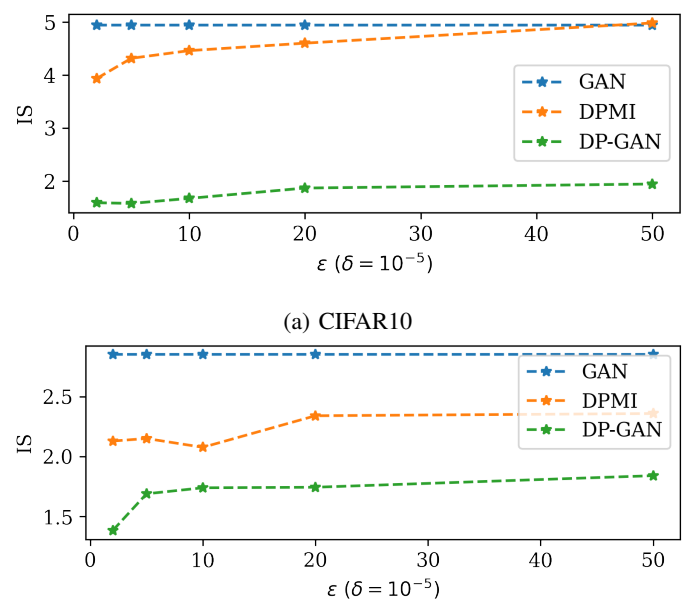

(b) SVHN

Fig. 7. The Inception Score across different datasets with different $\epsilon$.

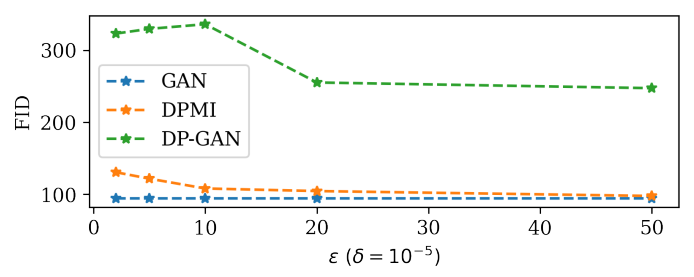

(a) CIFAR10

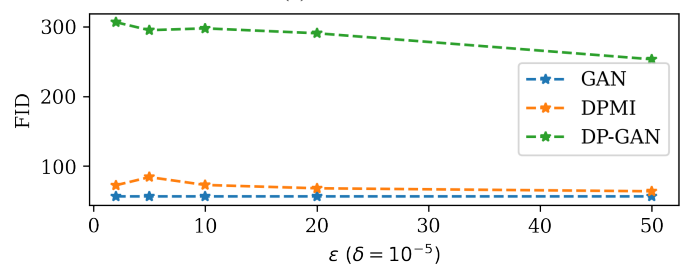

(b) SVHN

Fig. 8. The Frechet Inception Distance across different datasets with different $\epsilon$.

\section{ACKNOWLEDGEMENTS}

Research reported in this publication was supported by the National Institutes of Health, United States of America under award number R01MH121344-01 and the Child Family Endowed Professorship.

\section{REFERENCES}

[1] C. Dwork, A. Roth et al., "The algorithmic foundations of differential privacy." Foundations and Trends in Theoretical Computer Science, vol. 9, no. 3-4, pp. 211-407, 2014.
[2] I. Goodfellow, J. Pouget-Abadie, M. Mirza, B. Xu, D. Warde-Farley, S. Ozair, A. Courville, and Y. Bengio, "Generative adversarial nets," in Advances in neural information processing systems, 2014, pp. 26722680.

[3] P. Isola, J.-Y. Zhu, T. Zhou, and A. A. Efros, "Image-to-image translation with conditional adversarial networks," in Proceedings of the IEEE conference on computer vision and pattern recognition, 2017, pp. 11251134.

[4] H. Zhang, I. Goodfellow, D. Metaxas, and A. Odena, "Self-attention generative adversarial networks," in International conference on machine learning. PMLR, 2019, pp. 7354-7363.

[5] D. Pathak, P. Krahenbuhl, J. Donahue, T. Darrell, and A. A. Efros, "Context encoders: Feature learning by inpainting," in Proceedings of the IEEE conference on computer vision and pattern recognition, 2016, pp. 2536-2544.

[6] L. Fan, "A survey of differentially private generative adversarial networks," in The AAAI Workshop on Privacy-Preserving Artificial Intelligence, 2020.

[7] C. Esteban, S. L. Hyland, and G. Rätsch, "Real-valued (medical) time series generation with recurrent conditional gans," arXiv preprint arXiv:1706.02633, 2017.

[8] R. Shokri, M. Stronati, C. Song, and V. Shmatikov, "Membership inference attacks against machine learning models," in 2017 IEEE Symposium on Security and Privacy (SP). IEEE, 2017, pp. 3-18.

[9] M. Fredrikson, S. Jha, and T. Ristenpart, "Model inversion attacks that exploit confidence information and basic countermeasures," in Proceedings of the 22nd ACM SIGSAC Conference on Computer and Communications Security, 2015, pp. 1322-1333.

[10] J. Jordon, J. Yoon, and M. van der Schaar, "Pate-gan: Generating synthetic data with differential privacy guarantees," in International Conference on Learning Representations, 2018.

[11] D. Chen, T. Orekondy, and M. Fritz, "Gs-wgan: A gradient-sanitized approach for learning differentially private generators," Advances in Neural Information Processing Systems, vol. 33, 2020.

[12] M. Abadi, A. Chu, I. Goodfellow, H. B. McMahan, I. Mironov, K. Talwar, and L. Zhang, "Deep learning with differential privacy," in Proceedings of the 2016 ACM SIGSAC Conference on Computer and Communications Security, 2016, pp. 308-318.

[13] L. Xie, K. Lin, S. Wang, F. Wang, and J. Zhou, "Differentially private generative adversarial network," arXiv preprint arXiv:1802.06739, 2018.

[14] C. Xu, J. Ren, D. Zhang, Y. Zhang, Z. Qin, and K. Ren, "Ganobfuscator: Mitigating information leakage under gan via differential privacy," IEEE Transactions on Information Forensics and Security, vol. 14, no. 9, pp. 2358-2371, 2019.

[15] R. Torkzadehmahani, P. Kairouz, and B. Paten, "DP-CGAN: Differentially private synthetic data and label generation," in Proceedings of the IEEE Conference on Computer Vision and Pattern Recognition Workshops, 2019, pp. 0-0.

[16] S. Augenstein, H. B. McMahan, D. Ramage, S. Ramaswamy, P. Kairouz, M. Chen, R. Mathews et al., "Generative models for effective $\mathrm{ml}$ on private, decentralized datasets," arXiv preprint arXiv:1911.06679, 2019.

[17] B. K. Beaulieu-Jones, Z. S. Wu, C. Williams, R. Lee, S. P. Bhavnani, J. B. Byrd, and C. S. Greene, "Privacy-preserving generative deep neural networks support clinical data sharing," Circulation: Cardiovascular Quality and Outcomes, vol. 12, no. 7, p. e005122, 2019.

[18] B. Neyshabur, S. Bhojanapalli, and A. Chakrabarti, "Stabilizing gan training with multiple random projections," arXiv preprint arXiv:1705.07831, 2017.

[19] M. Arjovsky, S. Chintala, and L. Bottou, "Wasserstein gan," arXiv preprint arXiv:1701.07875, 2017.

[20] I. Gulrajani, F. Ahmed, M. Arjovsky, V. Dumoulin, and A. C. Courville, "Improved training of wasserstein gans," in Advances in neural information processing systems, 2017, pp. 5767-5777. 
[21] I. Mironov, "Rényi differential privacy," in 2017 IEEE 30th Computer Security Foundations Symposium (CSF). IEEE, 2017, pp. 263-275.

[22] S. Shalev-Shwartz and S. Ben-David, Understanding machine learning: From theory to algorithms. Cambridge university press, 2014.

[23] A. Krizhevsky, G. Hinton et al., "Learning multiple layers of features from tiny images," 2009.

[24] Y. Netzer, T. Wang, A. Coates, A. Bissacco, B. Wu, and A. Y. Ng, "Reading digits in natural images with unsupervised feature learning," 2011.

[25] S. Ozonoff, A.-M. Iosif, F. Baguio, I. C. Cook, M. M. Hill, T. Hutman, S. J. Rogers, A. Rozga, S. Sangha, M. Sigman et al., "A prospective study of the emergence of early behavioral signs of autism," Journal of the American Academy of Child \& Adolescent Psychiatry, vol. 49, no. 3, pp. 256-266, 2010.

[26] A. Borji, "Pros and cons of gan evaluation measures: New developments," arXiv preprint arXiv:2103.09396, 2021. 